\title{
Targeting Dynamin 2 as a Novel Pathway to Inhibit Cardiomyocyte Apoptosis Following Oxidative Stress
}

\author{
Danchen Gao Jian Yang ${ }^{\mathrm{a}}$ Yutao Wua ${ }^{\mathrm{a}}$ Qiwen Wang ${ }^{\mathrm{a}}$ Qiaoling Wang ${ }^{\mathrm{b}}$ En Yin Lai ${ }^{\mathrm{b}}$ \\ Jianhua Zhu \\ aDepartment of Cardiology, the First Affiliated Hospital, College of Medicine, Zhejiang University, \\ Hangzhou, 'Department of Physiology, College of Medicine, Zhejiang University, Hangzhou, Zhejiang, \\ China
}

\section{Key Words}

Dynamin $2 \cdot$ Oxidative stress $\bullet$ Ischemia/reperfusion injury $\bullet$ Apoptosis $\bullet$ mPTP

\begin{abstract}
Background/Aims: Inhibition of Drp-1-mediated mitochondrial fission limits reactive oxygen species (ROS) production and apoptosis in cardiomyocytes subjected to ischemia/reperfusion injury. It remains unknown if Dynamin 2 inhibition results in similar protective effects. Here we studied the role of Dynamin 2 in cardiomyocyte oxidative stress-induced apoptosis and ROS production. Methods: The effect of lentiviral shRNA (Iv5-shRNA) mediated Dynamin 2 knockdown on apopotosis, mitochondria, and ROS production were studied in neonatal mouse cardiomycytes, which were further treated with either selective Drp1 inhibitor mdivi-1 or the Dynamin 2/Drp1 inhibitor Dynasore. Apoptosis was evaluated by flow cytometry. Mitochondrial morphology and transmembrane potential $(\Delta \Psi \mathrm{m})$ were studied by confocal microscopy, and ROS production was detected by dichlorofluorescein diacetate. Results: Inhibition of Drp1 and Dynamin 2 protected against mitochondrial fragmentation, maintained $\Delta \Psi \mathrm{m}$, attenuated cellular ROS production and limited apoptosis. Moreover, Lv5-shRNA mediated knockdown of Dynamin 2 alleviated mitochondrial fragmentation, and reduced both ROS production and oxidative stress-induced apoptosis. The protective effects of Dynamin 2 knockdown were enhanced by Dynasore, indicating an added benefit. Conclusions: Oxidative stress-induced apoptosis and ROS production are attenuated by not only Drp1 inhibition but also Dynamin 2 inhibition, implicating Dynamin 2 as a mediator of oxidative stress in cardiomyocytes.
\end{abstract}

\section{Introduction}

Recently, it has been found that the fine balance between mitochondrial fusion and fission controlled by local GTP gradients and that mitochondrial energetics may determine the cell fate $[1,2]$. This balance can be disrupted by a variety of factors, including oxidative 
stress and simulated ischemia which predispose the cell to apoptosis [3].Stress and ischemia also open of the mitochondrial permeability transition pore (mPTP), which mediates of ischemia/reperfusion injury (IRI) [4]. Previous research has shown that mdivi-1, a smallmolecule inhibitor of Dynamin related protein 1(Drp1), significantly reduced the infarct size and IRI [5]. Also, inhibition of mitochondrial fission mediated by Drp1 can limit reactive oxygen species (ROS) production and apoptosis [6].

The large GTPase Dynamin, which has two forms Dynamin 1 and Dynamin 2, is the fission protein essential for clathrin-coated endocytic vesicle formation and release [7]. Dynamin 1 is highly expressed in the brain, whereas Dynamin 2 is expressed ubiquitously in organs including the heart [7]. The mitochondrial localized protein Drp1 is also a Dynamin protein and is critical for fission of the mitochondrial network [3]. Dynasore is a cell-permeable small molecule that inhibits the GTPase activity of Dynamin 1, Dynamin 2, and Drp1 [8, 9]. Our previous experiments revealed the protective effects of Dynasore against IRI in mice [9], likely through the inhibition of Drp1-mediated mitochondrial fission. Given that Dynamin 2 is highly expressed in cardiomyocytes [7] and a Dynamin 2 mutation is also associated with abnormal mitochondrial function [10], we asked whether Dynamin 2 inhibition is protective of mitochondria during oxidative stress, limits ROS production, and reduces cell apoptosis. We were interested if Dynasore mediates cardioprotective effect in ischemic/reperfused hearts.

Our results indicate that, in neonatal cardiomyocytes, Lv5-shRNA mediated Dynamin 2 knockdown significantly decreases ROS production and apoptosis after oxidative stress. In support of mitochondrial protection, we found that Dynamin 2 knockdown by Lv5shRNA was sufficient to prevent oxidative stress-induced mitochondrial fission in neonatal cardiomoyocytes, and the protective effect was further enhanced by Dynasore. Thus a small molecule based inhibitor of Dynamin 2 and Drp1 is a potential therapeutic approach for IRI.

\section{Materials and Methods}

\section{Guidelines for animal research}

This study and all procedures used in this study were approved by the Zhejiang University Medical School Committee on the Use and Care of Animals. Animals were housed in Zhejiang University facility of the Laboratory Animal Resource Center. All investigations conformed to the Guide for the Care and Use of Laboratory Animals published by the China National Institutes of Health.

\section{Materials}

Mdivi-1 and DRP1 Antibody (sc-32898) were purchased from Santa Cruz, MitoTracker was purchased from Invitrogen, Goat Anti-Rabbit IgG H\&L (HRP) (ab6721) and Anti-Dynamin 2 antibody (ab3457) were purchased from Abcam and beta Actin Antibody(20536-1-AP) was purchased from Proteintech. Unless otherwise stated, Dynasore, TMRM and all other materials were purchased from Sigma Chemical (St. Louis, MO). Mdivi-1 and Dynasore were dissolved in DMSO, with a final concentration of $0.1 \%$ DMSO.

\section{Isolation and culture of neonatal mouse cardiomyocytes}

Neonatal mouse cardiomyocytes were isolated from the ventricles of 1- to 3-day-old C57BL/6 mouse as previously described [11]. After plating, cardiomyocytes were replenished with fresh medium and subjected to drug treatment at $1 \mu \mathrm{M}, 3 \mu \mathrm{M}, 10 \mu \mathrm{M}$ Dynasore for 1 hour, $50 \mu \mathrm{M}$ mdivi-1 for 40 mins or vehicle followed by oxidative stress $\left(30 \mu \mathrm{M} \mathrm{H}_{2} \mathrm{O}_{2}\right)$ for $30 \mathrm{~min}$.

Proteins expression of Dynamin 2 and Drp1

Cell protein was isolated using the provided cell lysis buffer. Protein concentrations were measured using a bicinchoninic acid (BCA) protein assay kit (Beyotime Institute of Biotechnology, Haimen, China). Each sample was loaded separately on $12 \%(\mathrm{v} / \mathrm{v})$ sodium dodecyl sulfate-polyacrylamide gel electrophoresis (SDS-PAGE) and transferred to a polyvinylidene difluoride (PVDF) membrane. Membranes were blocked with 5\% (w/v) non-fat milk in Tween/TBS (TBST) for $1 \mathrm{~h}$ at room temperature and incubated with anti-Drp1, 


\section{Cellular Physiology Cell Physiol Biochem 2016;39:2121-2134 \begin{tabular}{l|l|l} 
and Biochemistry & Dublished online: November 02, 2016 & $\begin{array}{l}\text { (c) 2016 The Author(s). Published by S. Karger AG, Basel } \\
\text { www.karger.com/cpb }\end{array}$
\end{tabular} \\ Gao et al.: Targeting Dynamin 2}

anti-Dynamin 2 antibody (both at 1:500), or anti- $\beta$-actin (1:2000) in TBST overnight at $4^{\circ} \mathrm{C}$. Membranes were washed thrice with TBST and incubated with secondary antibody (horseradish peroxidase-conjugated, 1:2500) at room temperature for $1 \mathrm{~h}$. After washing 4-5 times with TBST, proteins were visualized with an enhanced chemiluminescence (ECL) detection reagent (Beit Haemek, Kibbutz Beit Haemek, Israel).

\section{Preparation of primary neonatal mouse cardiomyocyte Lv5-shRNA}

Packaging, purification, and titer determination of the lentivirus were performed as previously described [12]. The recombinant viruses were produced by calcium phosphate transfection of HEK293T cells using standard protocols [13]. Briefly, 293T cells were cultured in Dulbecco's modified Eagles's medium. The cells were co-transfected with the lentiviral vector $(10 \mu \mathrm{g})$, and the lentiviral packaging vectors pRSVREV ( $2 \mu \mathrm{g}), \mathrm{pMDLg} / \mathrm{RRE}(5 \mu \mathrm{g})$, and the vesicular stomatitis virus G glycoprotein (VSVG) expression vector pMD2G $(3 \mu \mathrm{g})$. The viruses were collected from the culture supernatant $48 \mathrm{~h}$ post-transfection, concentrated by ultra-centrifugation for $2 \mathrm{~h}$ at 25,000 rpm, and re-suspended in phosphate-buffered saline (PBS). Titers were determined by infecting $293 \mathrm{~T}$ cells with serial dilutions of concentrated lentivirus and then counting eGFP positive cells after $48 \mathrm{~h}$ under fluorescent microscopy. Lentiviral transduction in mouse neonatal cardiomyocytes: At day 2-3 post-culture, the cells were incubated with lentivirus at various "multiplicity of infection" (MOI) for 3, 4, and 5 days, respectively. Polybrene $(10 \mu \mathrm{g} / \mathrm{ml})$ was added to increase the efficiency of lentiviral transduction [14]. Neonatal mouse cardiomyocytes were infected with lentivirus transducing negative control shRNA (Lv5-shRNA ${ }_{N C}$ ) or Dynamin 2 lentivirus shRNA (Lv5-shRNA ${ }_{D 2}-1$ or Lv5-shRNA ${ }_{D 2}-2$ ) for $72 \mathrm{~h}$ and processed for immunoblot analysis. Immunoblot analysis was visualized with anti-Dynamin 2 or anti- $\beta$-actin antibodies. For all other experiments, only the control lv5-shRNA ${ }_{N C}$ and the more effective lv5-shRNA ${ }_{D 2}-2$ were used.

\section{Measurement of apoptosis in neonatal mouse cardiomyocytes}

Cell apoptosis was measured by flow cytometry using propidium iodide (PI) and Annexin-V staining. Annexin-V-only positive cells indicated early apoptosis, whereas PI-positive cells indicated damaged cell membranes and secondary/late apoptosis. For the assay, cells were dissociated using trypsin and then re-suspended in buffer to a final concentration of $1 \times 106$ cells/L. Each cell sample was incubated with annexin-V $(5 \mu \mathrm{L})$ and PI $(4 \mu \mathrm{l})$ in the dark at room temperature for 20 minutes and then analyzed using a FC500 MPL flow cytometer (Beckman Coulter, FL). The results were analyzed using FlowJo Software (TreeStar, Shenzhen, China) [15].

\section{Analysis of live-cell mitochondrial morphology}

Mitochondrial microscopy was performed by visualizing mitochondria using confocal fluorescence microscopy (Leica TCS SP5II, Germany) as previously described [16]. We selected 500nM as a working concentration for MitoTracker@ Red probe. After cardiomyocytes were stained with MitoTracker® Red CMXRos (500nM) for 30min, cells were pretreated with either control, Dynasore or mdivi-1 in indicated doses for specific time duration before being exposed to normal condition or $30 \mu \mathrm{M} \mathrm{H}_{2} \mathrm{O}_{2}$ for $30 \mathrm{~min}$. Before and after exposure to $\mathrm{H}_{2} \mathrm{O}_{2}$, cells were visualized using confocal fluorescence microscopy (Ex (nm) *: 579, Em (nm) *: 599) and a high resolution Cool SNAP HQ2 camera.

\section{Measurement of mitochondrial transmembrane potential $\Delta \Psi m$}

Quantification of mitochondrial membrane depolarization was carried out by measuring the fluorescence intensity of the membrane-permeant cationic probe tetramethylrhodamine methyl ester (TMRM). TMRM is a cationic fluorescent dye that accumulates inside the mitochondrial matrix by the membrane potential. The resulting signal of TMRM-stained cells thus represents only the probe accumulated in the intact mitochondria. A collapse on mitochondrial membrane is indicated by a decrease in fluorescence output. Induction of the mPTP opening is seen by collapse of the mitochondrial membrane potential $(\Delta \Psi \mathrm{m})$ $[17,18] . \Delta \Psi \mathrm{m}$ was measured with the non-quenching fluorescence methods (Molecular Probes, Sigma). Neonatal mouse cardiomyocytes were stained with TMRM $(600 \mathrm{nM})$ for $20 \mathrm{~min}$ at $37^{\circ} \mathrm{C}$ in the dark. Then cells were stained with TMRM $(150 \mathrm{nM})$ for $10 \mathrm{~min}$ at $37^{\circ} \mathrm{C}$ in the dark. The cells were then washed twice with PBS for $10 \mathrm{~min}$ each time. Cells were pretreated with the same protocols as previously mentioned. Cells were visualized using confocal fluorescence microscopy (Leica TCS SP5II, Germany), and a high resolution Cool SNAP HQ2 camera. For TMRM fluorescence, the cells were illuminated by using the 543-nm emission 


\section{Cellular Physiology Cell Physiol Biochem 2016;39:2121-2134 \begin{tabular}{ll|l} 
and Biochemistry & DOI: 10.1159/000447908 & (c) 2016 The Author(s). Published by S. Karger AG, Basel \\
www.karger.com/cpb
\end{tabular} \\ Gao et al.: Targeting Dynamin 2}

line of a He-Ne laser, and the fluorescence of TMRM was collected using a 570-nm long-pass filter. mPTP opening was confirmed in this model by using the known mPTP inhibitors mdivi-1.

Measurement of mitochondrial ROS production in neonatal mouse cardiomyocytes

Total ROS production in neonatal mouse cardiomyocytes exposed to oxidative stress was determined using dichlorofluorescein diacetate (DCFDA) fluorescence methods [15]. Briefly, cells were loaded with 10 $\mu \mathrm{M}$ (DCFDA) for $45 \mathrm{~min}$. ROS levels were measured using Fluoromax-3 spectrofluorimeter with excitation wavelength set at $485 \mathrm{~nm}$ and emission at $530 \mathrm{~nm}$, and it was quantified as a change in the fluorescence because of the conversion of non-fluorescent dichlorofluorescein diacetate to the highly fluorescent compound 2',7'-dichlorofluorescein (DCF) in the cells. Aliquots of cell suspension (100 $\mu$ l) were incubated for 10 min with curcumin and re-suspended in HBS prior to each experiment, which potentially eliminated the interference of curcumin in fluorescence assays.

Data analysis

Statistical analysis was performed using SPSS16.0. The data were presented as means \pm standard deviation. Difference between control and experimental groups were determined using a one-way analysis of variance (ANOVA) for multiple groups. Difference between every two groups was assessed using Bonferroni post hoc test. For comparison between two groups with timed repeated measurements, a twoway ANOVA (treatment and time were considered as the two variances) was used. $\mathrm{P}<0.05$ was considered to be statistically significant.

\section{Results}

Dynamin 2 knockdown reduces hydrogen peroxide-induced apoptosis in neonatal mouse cardiomyocytes

Our previous study found that Dynasore protects adult mouse cardiomyocytes against oxidative stress, and dramatically reduced infarct size (from 39.6\% to 8.1\%, near $80 \%$ reduction) in Langendorff perfused adult mouse hearts following 30 minutes global ischemia and 60 minutes of reperfusion [9]. In the same adult mouse I/R model, we further identified that, as compared to DMSO control, $1 \mu \mathrm{M}$ Dynasore pretreatment prevented the elevation of LVEDP [9], achieved significant recoveries of $-\mathrm{dp} / \mathrm{dt}$ and $+\mathrm{dp} / \mathrm{dt}$, and decreased the Tau $(\mathrm{p}<0.05)$ (Data not shown). In addition to Drp1, Dynasore also inhibits the GTPase activity of Dynamin 2, mutation of which is associated with abnormal mitochondrial function [10]. Thus Dynamin 2 inhibition may alter mitochondrial related apoptosis, contributing to the significant cardioprotective effect by low dose Dynasore. Given it is not possible to study apoptosis in isolated adult cardiomyocytes which only survive 3 days in culture, we used neonatal mouse cardiomyocytes to study the effect of Dynamin 2 knockdown on cell apoptosis following oxidative stress. Neonatal mouse cardiomyocytes were treated with Lv5-shRNA ${ }_{D 2}-1$ or Lv5-shRNA ${ }_{D 2}-2$ to specifically knock down Dynamin 2 and compared to a non-targeting negative control Lv5-shRNA ${ }_{N C}$ (shRNA-NC). As indicated in Fig. 1A, Lv5shRNA $_{D 2}-2$ was the effective shRNA in knocking down Dynamin 2, which induced a near $70 \%$ decrease in the protein expression of Dynamin 2 (to 34.7\%) by Western blotting. Thus, Lv5shRNA $_{D 2}-2$ was used in the following experiments in the current study. After knockdown, neonatal cardiomyocytes were subjected to $\mathrm{H}_{2} \mathrm{O}_{2}$ treatment and apoptosis was then analyzed by flow cytometry (Fig. 1B, quantification in Fig. 1C). Our results indicated that apoptosis was significantly decreased in Lv5-shRNA ${ }_{D 2}-2$ treated cardiomyocytes $(28.09 \%$ vs $44.1 \%, \mathrm{P}<0.01$ ) similar to the effect of Drp1 inhibition by mdivi-1. Between the Lv-shRNA ${ }_{D 2}-2$ and Lv-shRNA ${ }_{N C}$ treated groups, the apoptosis rate was $6.78 \%$ vs $9.97 \%(\mathrm{p}<0.001)$ with $10 \mu \mathrm{M}$ Dynasore pretreatment, and $14.67 \%$ vs $14.9 \%$ ( $p>0.05$ ) with $50 \mu \mathrm{M}$ mdivi-1 pretreatment, respectively. The further decrease in oxidative stress-induced apoptosis after higher doses of Dynasore pretreatment indicates an additive effect of Dynamin 2 inhibition to Drp1 inhibition. These results indicate that Dynamin 2 is involved in cardiomyocyte apoptosis and cell survival. Apoptosis assay standardization was done (Data not shown). 


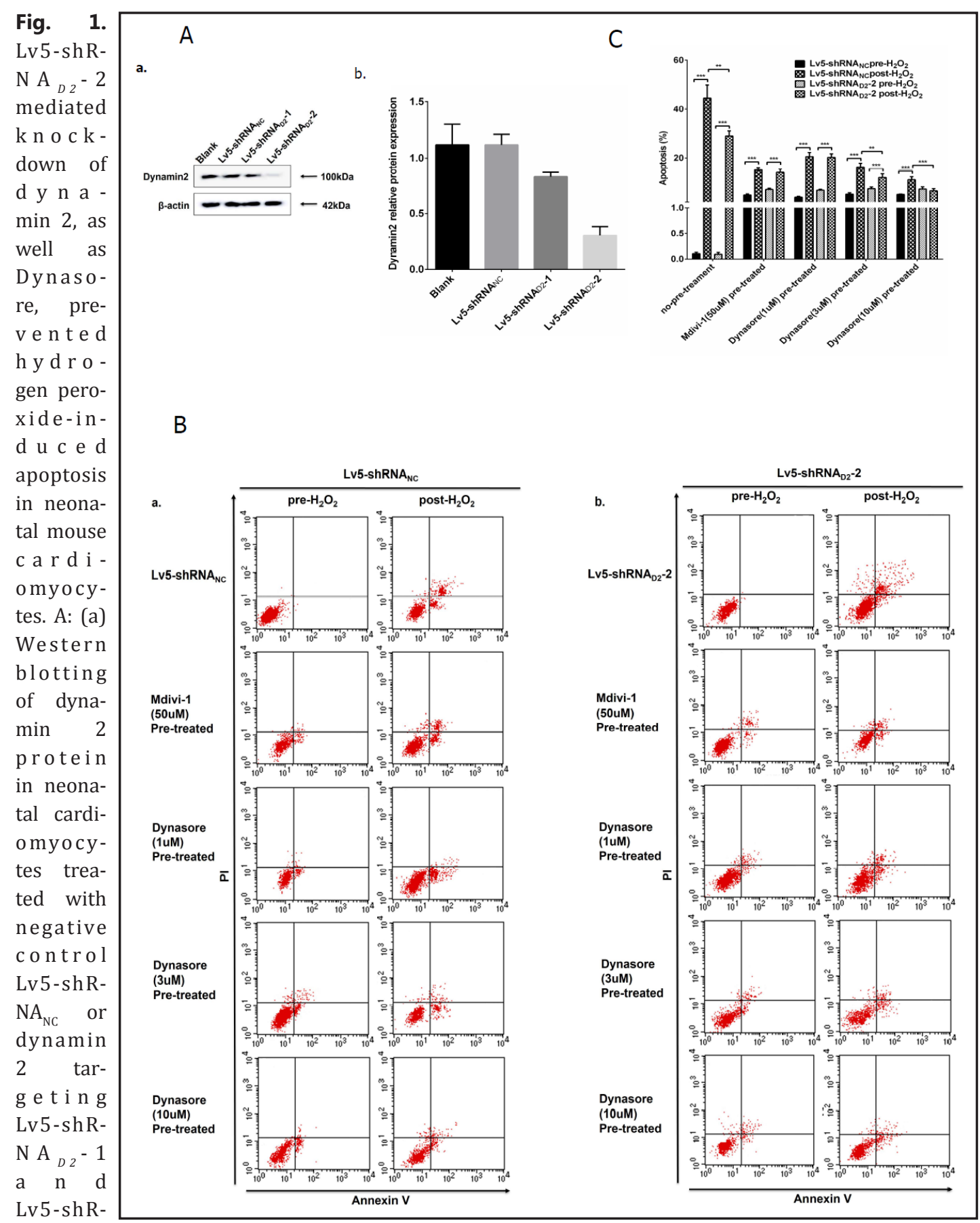

$\mathrm{NA}_{D 2}$-2. Lv5-shRNA ${ }_{D 2}-2$ induces a near $70 \%$ reduction in dynamin 2 protein as indicated in the quantification data included in the bar graph (b) . B: Both shRNA-mediated dynamin2 knockdown and Dynasore diminish the apoptotic hallmarks in the neonatal mouse cardiomyocytes. (a) The cardiomyocytes-shRNA $N C$ were pre-treated with Mdivi-1 $(50 \mu \mathrm{M}) /$ Dynasore $(1 \mu \mathrm{M}) /$ dynasore $(3 \mu \mathrm{M}) /$ dynasore $(10 \mu \mathrm{M})$ respectively before being exposed to $\mathrm{H}_{2} \mathrm{O}_{2}(30 \mu \mathrm{M})$ for $30 \mathrm{~min}$. (b) The cardiomyocytes- shRNA ${ }_{D 2}-2$ were pre-treated with Mdivi-1 $(50 \mu \mathrm{M})$ / Dynasore $(1 \mu \mathrm{M}) /$ dynasore $(3 \mu \mathrm{M}) /$ dynasore $(10 \mu \mathrm{M})$ respectively before being exposed to $\mathrm{H}_{2} \mathrm{O}_{2}(30 \mu \mathrm{M})$ for $30 \mathrm{~min}$. C: The bar graph shows quantification of apoptosis, which The $\%$ of apoptotic cells means the sum \%-values of quadrants Q2 and Q4 from 1B. Cells were stained with annexin-V (AnxV) and PI to be distinguished by flowcytometry for unaffected cells (AnxV ${ }^{\text {neg }} \mathrm{PI}^{\text {neg }}$ ), early apoptotic cells (AnxV $V^{\text {po- }}$ ${ }^{s} \mathrm{PI}^{\mathrm{neg}}$ ), and cells in late apoptosis $\left(\mathrm{AnxV}^{\mathrm{pos}} \mathrm{PI}^{\mathrm{pos}}\right)$. Each result shown is representative of five experiments. All data are expressed as mean $\pm \mathrm{SD}$. ${ }^{* *} \mathrm{P}<0.01 ;{ }^{* * *} \mathrm{P}<0.001$. 
A

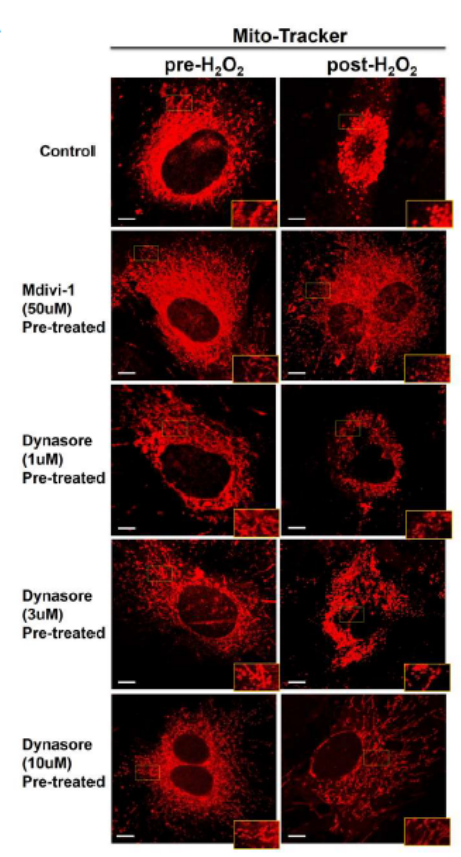

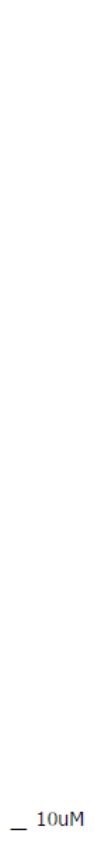

C

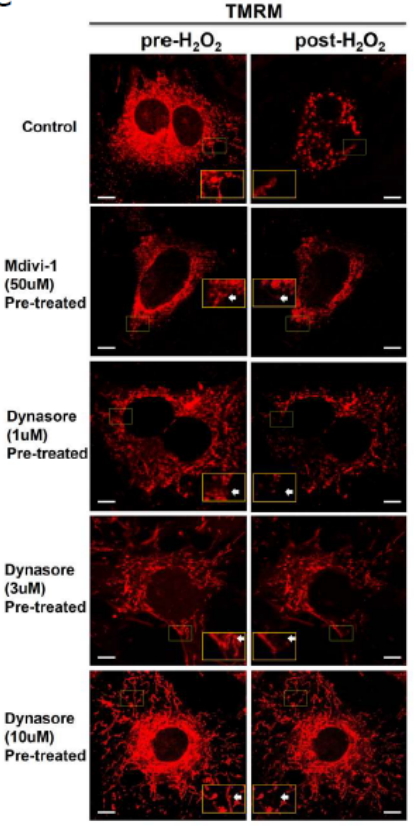

B

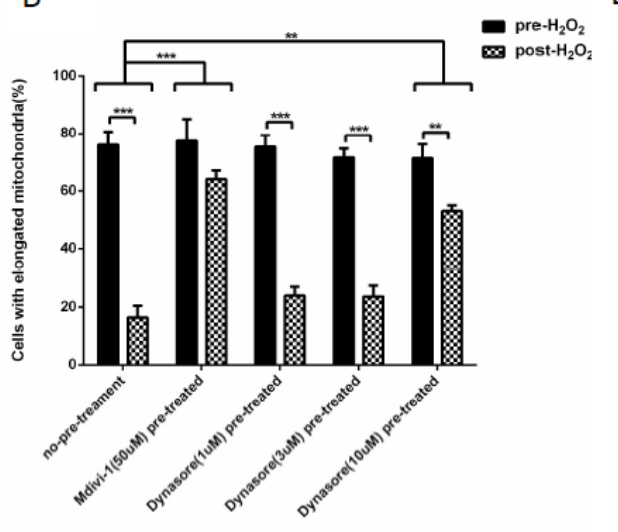

D

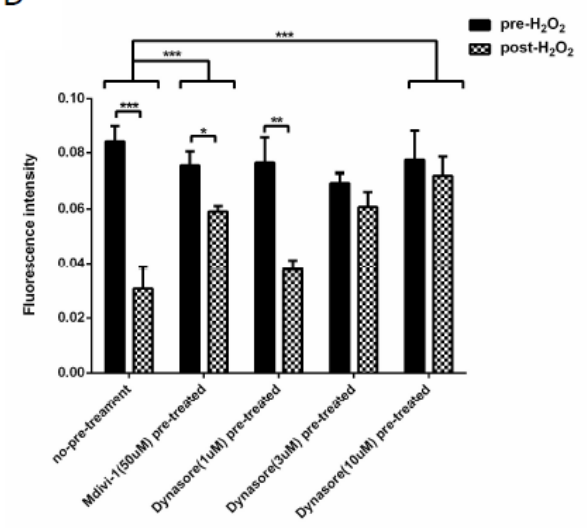

Fig. 2. Dynasore prevents oxidative stress-induced changes in mitochondrial morphology and $\Delta \Psi \mathrm{m}$. A: Representative confocal mitochondrial morphology fluorescence images depicting neonatal mouse cardiomyocytes subjected to oxidative stress. The cells were loaded with MitoTracker® Red (500nM), then pre-treated with Mdivi-1 $(50 \mu \mathrm{M}) /$ dynasore $(1 \mu \mathrm{M}) /$ Dynasore $(3 \mu \mathrm{M}) /$ Dynasore $(10 \mu \mathrm{M})$ respectively before being exposed to $\mathrm{H}_{2} \mathrm{O}_{2}(30 \mu \mathrm{M})$ for $30 \mathrm{~min}$. The bottom inserts are the higher power view of the boxed areas in the same panel. Scale bar: 10um . B: The bar graph shows the percentage of neonatal mouse cardiomyocytes displaying elongated mitochondria using confocal images. $n=3$ experiments with 7 to 10 cells per treatment group. C: Representative confocal mitochondrial $\Delta \Psi \mathrm{m}$ fluorescence images depicting neonatal mouse cardiomyocytes subjected to oxidative stress. The cells were loaded with TMRM(150nM), then pre-treated with Mdivi-1 $(50 \mu \mathrm{M}) /$ Dynasore $(1 \mu \mathrm{M}) /$ Dynasore $(3 \mu \mathrm{M})$ / Dynasore $(10 \mu \mathrm{M})$ respectively before being exposed to $\mathrm{H}_{2} \mathrm{O}_{2}(30 \mu \mathrm{M})$ for 30min. The intensity of TMRM reflects the level of mitochondrial transmembrane potential. The bottom inserts are the higher power view of the boxed areas in the same panel. The arrow shows a mitochondrion that underwent a loss followed by a restoration of $\Delta \Psi \mathrm{m}$ with a simultaneous $\mathrm{H}_{2} \mathrm{O}_{2}$ exposure. Two sequential confocal images collected from a region of interest in single cell. Scale bar: 10um . D: The bar graph shows quantification of TMRM signals in the neonatal mouse cardiomyocytes subjected to oxidative stress. $n=3$ experiments with 20 cells per treatment group. All data are expressed as mean $\pm \mathrm{SD}$. ${ }^{*} \mathrm{P}<0.05 ;{ }^{* *} \mathrm{P}<0.01 ;{ }^{* * *} \mathrm{P}<0.001$.

\section{KARGER}




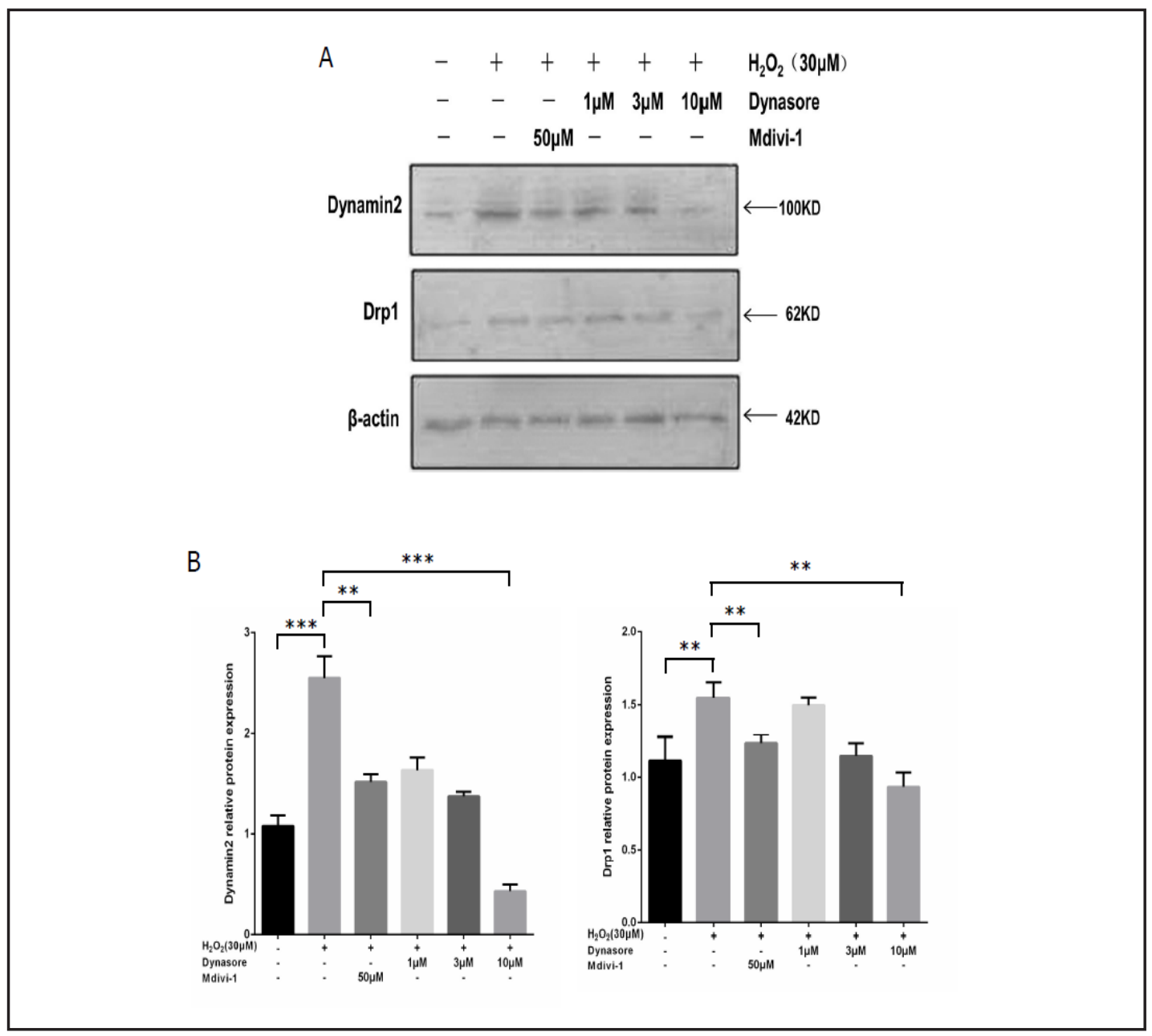

Fig. 3. Dynasore reduced the protein levels of Drp1 and dynamin 2 in the hydrogen peroxide-inflicted neonatal mouse cardiomyocytes. A: Western blot was used to evaluate the protein levels of Drp1 and Dynamin2 in cardiomyocytes subjected to oxidative stress. The cells were pre-treated with mdivi-1 (50 $\mu \mathrm{M}) /$ dynasore $(1 \mu \mathrm{M}) /$ dynasore $(3 \mu \mathrm{M}) /$ dynasore $(10 \mu \mathrm{M})$, respectively, before being exposed to $\mathrm{H}_{2} \mathrm{O}_{2}(30 \mu \mathrm{M})$ for $30 \mathrm{~min}$. The blot was probed with the indicated antibodies, revealing a target-specific protein reduction in comparison with untreated samples. B: Quantification of protein bands are included in the bar graph. Each result shown is representative of five experiments. All data are expressed as mean $\pm \mathrm{SD}$. ${ }^{* *} \mathrm{P}<0.01$; $* * * \mathrm{P}<0.001$.

Dynasore protects against oxidative stress-induced mitochondrial morphological damage and preserves transmembrane potential $\Delta \Psi \mathrm{m}$

Given that Drp1 inhibition protects against oxidative stress-induced mitochondrial fragmentation, we studied the effects of Dynasore and mdivi-1 on mitochondrial morphology by MitoTracker labeling and mitochondrial transmembrane potential $\Delta \Psi \mathrm{m}$ by TMRM labeling in neonatal mouse cardiomyocytes. Cardiomyocytes were confirmed by positive anti- $\alpha$ actin labeling. Consistent with previous observation in Hela cells [8], Dynasore prevented oxidative stress-induced mitochondrial fission, maintaining an elongated mitochondrial network (Fig. 2A, quantification in Fig. 2B). While $1 \mu \mathrm{M}$ Dynasore pretreatment slightly improved mitochondrial morphology, $10 \mu \mathrm{M}$ Dynasore significantly elongated mitochondria compared with control $(\mathrm{P}<0.01,<0.001$, respectively, Fig. 2B). Consistent with its known effect in inhibiting Drp1 [5], 50 $\mu \mathrm{M}$ mdivi-1 treatment significantly improved mitochondrial 
Fig. 4. Effects of silencing of dynamin 2 translation on $\mathrm{H}_{2} \mathrm{O}_{2}$-induced changes in mitochondrial morphology in neonatal mouse cardiomyocytes. A, B: Representative confocal mitochondrial morphology fluorescence images depicting cardiomyocytes-shRNA ${ }_{N C}(\mathrm{~A})$ and cardiomyocytes-shRNA ${ }_{D 2}-2$ (B) subjected to oxidative stress. The cells were loaded with MitoTracker $®$ Red(500nM), then pre-treated with Mdivi-1 $(50 \mu \mathrm{M})$ / Dynasore $(1 \mu \mathrm{M}) /$ Dynasore $(3 \mu \mathrm{M}) /$ Dynasore $(10 \mu \mathrm{M})$ respectively before being exposed to $\mathrm{H}_{2} \mathrm{O}_{2} \quad(30 \mu \mathrm{M})$ for $30 \mathrm{~min}$. The bottom inserts are the higher power view of the boxed areas in the same panel. Scale bar: 10um. C: The bar graph shows the percentage of cardiomyocytes-sh RNA ${ }_{N C}$ and cardiomyocytes- shR$\mathrm{NA}_{D 2}-2$ displaying elongated mitochondria using confocal images. $n=3$ experiments with 10 cells per treatment group. All data are expressed as mean $\pm \mathrm{SD}$. ${ }^{*} \mathrm{P}<0.05$; ${ }^{* *} \mathrm{P}<0.01 ;{ }^{* * *} \mathrm{P}<0.001$.

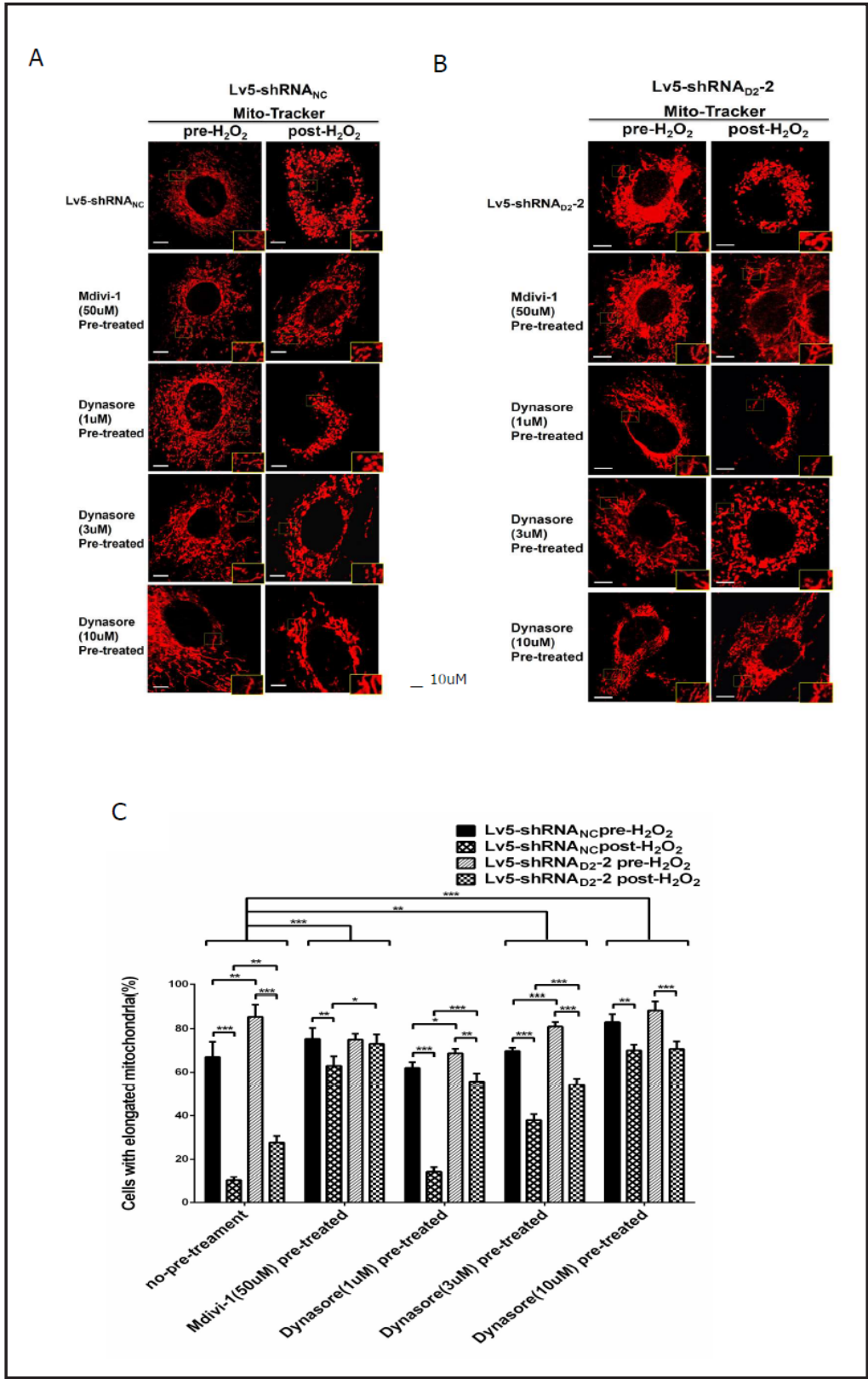

morphology. Results in Figure 2C indicate a significant loss of TMRM fluorescence, accompanied by a decrease in $\Delta \Psi \mathrm{m}$ after the cell exposure to $30 \mu \mathrm{M} \mathrm{H}_{2} \mathrm{O}_{2}$ for $30 \mathrm{~min}$. Similarly, $50 \mu \mathrm{M}$ mdivi-1 protected TMRM fluorescence and $\Delta \Psi \mathrm{m}$. Dynasore protected the mitochondrial transmembrane potential $\Delta \Psi \mathrm{m}$ in neonatal mouse cardiomyocytes as well (Fig. 2C, D). Furthermore, the beneficial effect of Dynasore on mitochondrial morphology and $\Delta \Psi \mathrm{m}$ was also dose-dependent $(\mathrm{P}<0.001$, Fig. $2 \mathrm{D})$. Interestingly, as compared to mdivi-1, Dynasore tended to have a greater effect on mitochondrial transmembrane potential. Since low dose Dynasore $(1 \mu \mathrm{M})$ is already sufficient to preserve cardiomyocyte survival [9], it is likely that, in addition to the inhibition of Drp1-mediated mitochondrial fission, the cardioprotective effect of Dynasore is mediated by its inhibition of another player, possibly Dynamin 2. The involvement of Dynamin 2 was further supported by a significant increase in Dynamin 2 protein levels following oxidative stress, which can be reduced by Dynasore $(\mathrm{P}<0.001$,Fig. 3). Similarly, Drp1 was also increased after oxidative stress $(\mathrm{P}<0.01$,Fig. 3$)$, which was reduced by both mdivi-1 and $10 \mu \mathrm{M}$ Dynasore $(\mathrm{P}<0.01, \mathrm{P}<0.001$,Fig. 3$)$. 


\section{Cellular Physiology \begin{tabular}{ll|l} 
and Biochemistry $10.1159 / 000447908$ & C) 2016 The Author(s). \\
Published online: November 02, 2016 & www.karger.com/cpb
\end{tabular}

Fig. 5. Effects of silencing of dynamin2 translation on $\mathrm{H}_{2} \mathrm{O}_{2}$-induced changes in mitochondrial $\Delta \Psi \mathrm{m}$ in neonatal mouse cardiomyocytes. A, B: Representative confocal mitochondrial $\Delta \Psi \mathrm{m}$ fluorescence images depicting neonatal mouse cardiomyocytes-shRNA $A_{N C}$ (A) and cardiomyocytes -shRNA ${ }_{D 2}-2$ (B) subjected to oxidative stress. The cells were loaded with TMRM (150nM), then pre-treated with Mdivi-1 $(50 \mu \mathrm{M}) /$ Dynasore $(1 \mu \mathrm{M}) /$ Dynasore $(3 \mu \mathrm{M}) /$ Dynasore $(10 \mu \mathrm{M})$ respectively before being exposed to $\mathrm{H}_{2} \mathrm{O}_{2}(30 \mu \mathrm{M})$ for $30 \mathrm{~min}$. The intensity of TMRM reflects the level of mitochondrial transmembrane potential. The bottom inserts are the higher power view of the boxed areas in the same panel. The arrow shows a mitochondrion that underwent a loss followed by a restoration of $\Delta \Psi \mathrm{m}$ with simultaneous exposure to $\mathrm{H}_{2} \mathrm{O}_{2}$. Two sequential confocal images collected from a region of interest in single cell. Scale bar: 10um. C: The bar graph shows quantification of TMRM signals in the cardiomyocytes-shRNA ${ }_{N C}$ and cardiomyocytes-shRNA $_{D 2}-2$ subjected to oxidative stress. $\mathrm{n}=3$ experiments with 20 cells per treatment group. All data are expressed as mean \pm SD. ${ }^{*} \mathrm{P}<0.05$; ${ }^{* *} \mathrm{P}<0.01 ;{ }^{* * *} \mathrm{P}<0.001$.

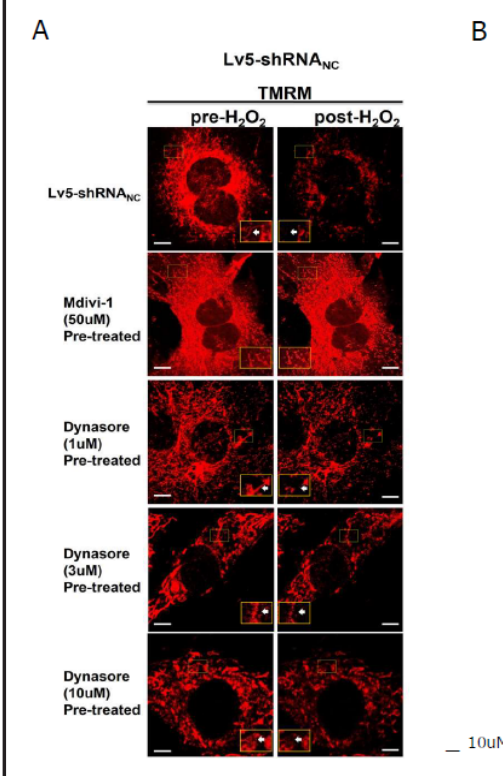

B

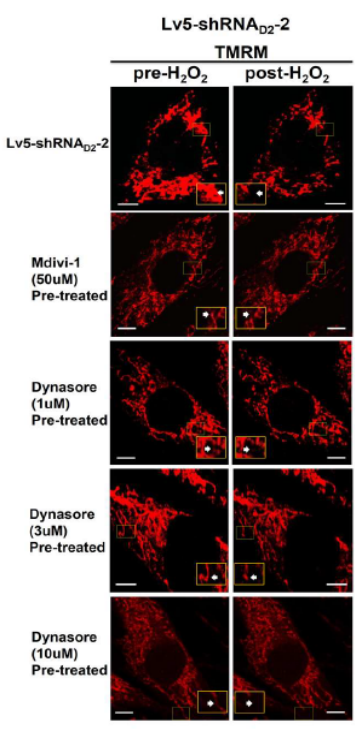

$\mathrm{C}$

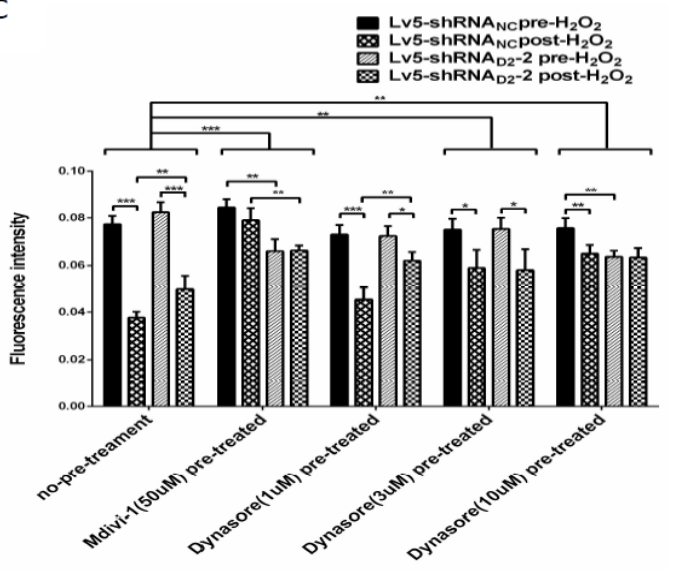

Dynamin 2 knockdown protects mitochondrial morphology and $\Delta \Psi m$ in stressed neonatal mouse cardiomyocytes

Because Dynamin 2 knockdown protects cell apoptosis which is linked to mitochondrial function (Fig. 1), it is logical to hypothesize that Dynamin 2 inhibition protects mitochondrial function. To test whether Dynamin 2 knockdown affects mitochondrial network, we then analyzed mitochondrial morphology in Lv5-shRNA ${ }_{D 2}-2$ treated neonatal mouse cardiomyocytes subjected to $\mathrm{H}_{2} \mathrm{O}_{2}$. Cardiomyocytes were then labeled with MitoTracker for analysis of mitochondrial morphology, or TMRM for measurement of mitochondrial

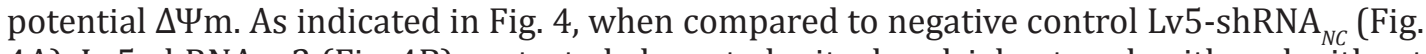
$4 \mathrm{~A}$ ), Lv5-shRNA ${ }_{D 2}-2$ (Fig. 4B) protected elongated mitochondrial network with and without exposure to oxidative stress ( $\mathrm{p}<0.01$, Fig. 4C). Consistently, in control Lv5-shRNA ${ }_{N C}$ treated cardiomyocytes, Dynasore elongated mitochondria in a dose-dependent fashion, with $10 \mu \mathrm{M}$ of Dynasore achieving the similar protective effect of mdivi-1 (Fig. 4A). As predicted, in cells with Dynamin 2 knockdown by Lv5-shRNA ${ }_{D 2}-2$, there was significant improvement of mitochondrial morphology in $1 \mu \mathrm{M}$ and $3 \mu \mathrm{M}$ Dynasore similar to the maximal beneficial effect provided by $10 \mu \mathrm{M}$ Dynasore and mdivi-1 in cardiomyocytes without Dynamin 2 
Fig. 6. Lv5-shRNA $A_{D 2}-2$ and Dynasore reduce the production of mitochondrial ROS in neonatal mouse cardiomyocytes. Conventional estimates of mitochondrial ROS (mtROS) of cardiomyocytes mitochondria under different experimental conditions, estimated by fluorescence intensity of 2',7'-dichlorofluorescein (DCF). $n=3$ experiments with 20 cells per treatment group. All data are expressed as mean \pm SD. ${ }^{*} \mathrm{P}<0.05$; ${ }^{*} \mathrm{P}<0.01$; $* * * \mathrm{P}<0.001$.

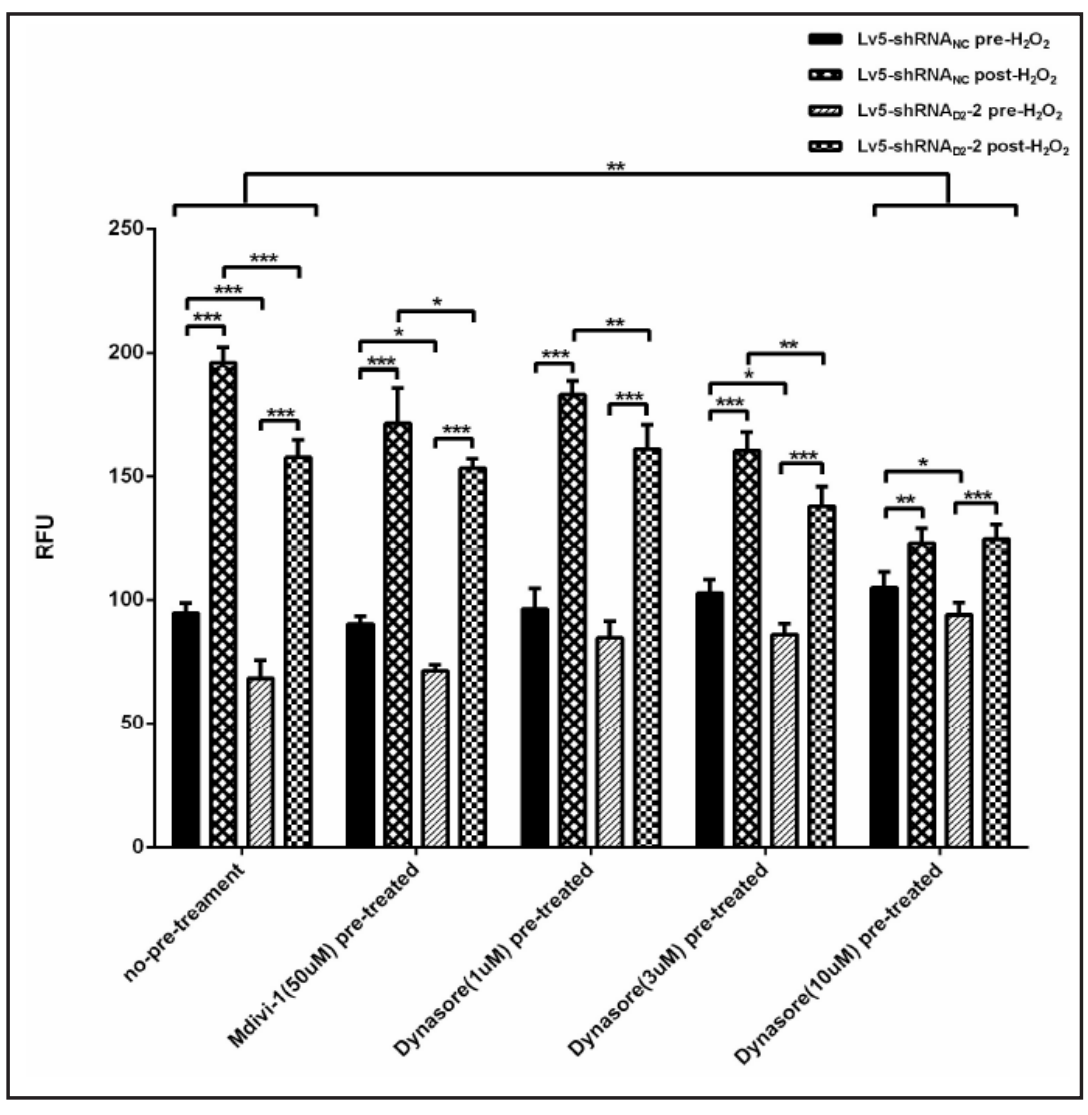

knockdown (Fig. 4C). Similar effects on protecting mitochondrial transmembrane potential $\Delta \Psi$ m were observed (Fig. 5). These results suggested that Dynamin 2 is involved in dynamic equilibrium between mitochondrial fusion and fission, contributing to the cardiomyocyte protective effect by low dose Dynasore.

Dynamin 2 knockdown reduces the production of mitochondrial ROS in neonatal mouse cardiomyocytes

ROS are generated in mitochondria as a result of the uncoupling of the electron transport chain which is a step in the intrinsic apoptotic pathway $[19,20]$. As a consequence, ROS is a common inducer of mPTP opening, promoting oxidative damage of cell macromolecules and leading to apoptosis in I/R injured myocardium [21,22]. We evaluated the effect of Dynasore on $\mathrm{H}_{2} \mathrm{O}_{2}$-induced intracellular ROS production (data not shown). Our results indicated that pre-treating cells with $1 \mu \mathrm{M}, 3 \mu \mathrm{M}, 10 \mu \mathrm{M}$ Dynasore and $50 \mu \mathrm{M}$ mdivi-1 significantly reduced $\mathrm{H}_{2} \mathrm{O}_{2}$-induced ROS generation, although no significant difference was observed among pretreatment groups with different doses of Dynasore and mdivi$1(\mathrm{P}>0.05)$. The data indicate that ROS reduction by low dose Dynasore is mediated through Dynamin 2 inhibition. Thus, we hypothesized that the cardioprotective effect of dynasore is mediated not only by inhibition of Drp1-dependent mitochondrial fission but also the inhibition of Dynamin 2-dependent ROS production. To test this hypothesis, we evaluated ROS production in neonatal cardiomyocytes with Dynamin 2 knockdown by Lv5-shRNA ${ }_{D 2}-2$. Our results indicate that oxidative stress increases intracellular ROS generation, while Lv5shRNA $_{D 2}-2$ treated cardiomyocytes reduces ROS production. Furthermore, with Dynamin 2 knockdown, Dynasore decreases ROS production, with a significant difference between the $10 \mu \mathrm{M}$ Dynasore group and untreated group $(\mathrm{P}<0.01 \mathrm{Fig} .6)$. However, there was no difference in the increase of levels of ROS production between the mdivi-1 treatment Lv5-shRNA and Lv5-shRNA ${ }_{D 2}-2$ groups ( $P>0.05$, Fig. 6). The results suggested that ROS production is dependent on Dynamin 2 activity. 


\section{Cellular Physiology Cell Physiol Biochem 2016;39:2121-2134 \\ \begin{tabular}{ll|l} 
and BiOChemistry & $\begin{array}{l}\text { DOI: 10.1159/000447908 } \\
\text { Published online: November 02, } 2016\end{array}$ & $\begin{array}{l}\text { C } 2016 \text { The Author(s). Published by S. Karger AG, Basel } \\
\text { www.karger.com/cpb }\end{array}$
\end{tabular} \\ Gao et al.: Targeting Dynamin 2}

\section{Discussion}

Drp1 inhibition is known to reduce cardiomyocyte death [5]. Combined with earlier studies [9], our data suggest a critical role of Dynasore in inhibiting Drp1 and mPTP opening in cardiomyocytes subjected to oxidative stress. Furthermore, our study is the first to reveal the role of Dynamin 2 in ROS production independent of the mitochondrial electron transport or xanthine/xanthine oxidase systems [23, 24], and Dynamin 2 knockdown is anti-apoptosis and pro-survival in neonatal mouse cardiomyocytes.

There are very few published studies on the protective effects of Dynamin 2 inhibition in decreasing mitochondrial fission and apoptotic cell death, and earlier studies have been confined to neurodegenerative or neoplastic diseases and tended to focus on the drug effect on endocytosis $[25,26]$. Here we identified that the Dynamin 2 inhibitor Dynasore significantly prevented oxidative stress-induced mitochondrial fission in neonatal mouse cardiomyocytes (Fig. 2), along with a decrease in cell apoptosis (Fig. 1). During ischemia, the dynamic balance shifts from fusion to fission, resulting in fragmented and discontinuous mitochondria [5, 27], as well as mitochondrial outer membrane permeablization, release of apoptotic factors, and activation of apoptosis. Taken together, our results indicated that Dynasore protects cultured primary mouse neonatal cardiomyocytes from oxidative stressinduced cell apoptosis. The underlying mechanism may be maintaining mitochondrial mPTP [28]. Interestingly, $10 \mu \mathrm{M}$ Dynasore and $50 \mu \mathrm{M}$ mdivi-1 both reduced neonatal cardiomyocyte apoptosis (Fig. 1). While the beneficial effects of Dynasore on survival were dose-dependent, there was a greater reduction of cell apoptosis with $10 \mu \mathrm{M}$ Dynasore pretreatment than $50 \mu \mathrm{M}$ mdivi-1 group (Fig. 1).

Our study reveals that the protein levels of Dynamin 2 and Drp1 increase significantly when cardiomyocytes were exposed to $30 \mu \mathrm{M} \mathrm{H}_{2} \mathrm{O}_{2}$, which can be significantly reduced by

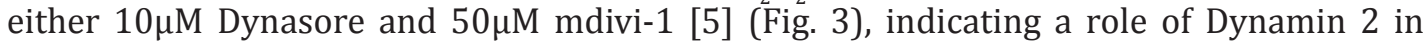
oxidative stress. Meanwhile, Dynasore produces a lower rate of apoptosis, indicating that Dynamin 2 is involved in cardiomyocyte apoptosis.

Although there is increasing evidence that Drp-1-involved mitochondrial fission is extensive in cardiomyocytes, our present study, to our knowledge, is the first to suggest that Dynamin 2 increases ROS production. The subsequent activation of fission proteinsand GTPase Drp1 enzymatic activity will ultimately affect the structural integrity of mitochondria, creating an imbalance in mitochondrial dynamics, and causing excessive mitochondrial fission, with vicious cycle of apoptosis and necrosis to follow. Our results also support previous observed alterations in Dynamin 2 following hypoxia and ischemia/reperfusion. For example, Singleton found hyperoxia treatment of human pulmonary artery endothelial cells induced recruitment of the vesicular regulator, Dynamin 2, the non-receptor tyrosine kinase, c-Abl, and the NADPH oxidase subunit, p47phox, to caveolin-enriched microdomains [29]. Zamora study suggested Nox as a major source of ROS production during ischemia/ reperfusion [30]. However, whether the Nox family is involved in ROS production outside of the mitochondria is yet to be defined.

Dynamin 2, in addition to its classical role leading to the scission of endocyte vesicles at the plasma membrane [31], has also been implicated in other cellular processes such as: regulation of actin assembly and reorganization through interacting with actin-binding proteins [32, 33]; disruption of the Dynamin 2-dependent retrograde trafficking of $\mathrm{Na}+\mathrm{H}+$ exchanger-1 contributes to cardiomyocyte apoptosis [34], and Dynamin 2 is a new connexin partner through an innovating mechanistic model by which Dynamin 2 may control connexin 43 gap junction plaque invagination, endocytosis, recycling and degradation [35]. In the present study, we demonstrated for the first time that Dynamin 2 was a new partner with oxidative stress protein, which may play a fundamental role during the process of oxidative injury. Fish et al reported that the p53 pathway might be mainly responsible for the apoptosis promoted by over expressed Dynamin 2, indicating Dynamin 2 as part of a signaling pathway that can activate the transcription factor p53 and potentially trigger apoptosis $[36,37]$. A recent study reported endocytosis has been implicated in the regulation of several signaling 
pathways, however, the current cardioprotection dose of Dynasore $(\leq 1 \mu \mathrm{M})$ is lower than the previously reported inhibitory dose of Dynasore (IC50 15 $\mu \mathrm{M}$ ) on Dynamin dependent endocytosis [38], indicating Dynamin 2 activity as a signaling GTPase can occur independent of any measurable effects on endocytosis [36].

Data from our study have shed light on the correlation between oxidative stress and Dynamin 2 in the maintenance and regulation of cardiomyocytes survival and cardiacfunction. However, it must be noted that the current study is limited to neonatal cells and the oxidative stress model of mPTP opening may not accurately reflect the conditions of ischemia and reperfusion in adult hearts, which should be further investigated in future studies. Further study is also needed to elucidate the precise mechanisms of Dynamin 2 inhibition against IRI and IRI-induced cardiac diastolic dysfunction. It would also be ideal to use selective inhibitors of Dynamin 2 rather than Dynasore in the future investigation.

In conclusion, oxidative stress-induced cell apoptosis can be attenuated by not only Drp1 inhibition but also Dynamin 2 inhibition, both of which suppress ROS production. Our study expands the scope of oxidative stress-triggered cellular responses in cardiomyocytes to include Dynamin 2 involvement.

\section{Abbreviations}

IRI (ischemia/reperfusion injury); ATP (adenosine triphosphate); ROS (reactive oxygen species); mPTP (mitochondrial permeability transition pore); MFN1 (mitofusin 1); MFN2 (mitofusin 2); OPA1 (optic atrophy protein 1); FIS1 (fission protein 1); MFF (mitochondrial fission factor); Drp1 (Dynamin related protein 1); LVEDP (Left ventricular end diastolic pressure); LVDP (Left ventricular developed pressure); LVESP (left ventricular end systolic pressure); BCA (bicinchoninic acid); TBE (trypan blue exclusion); TMRM (Tetramethylrhodamine methyl ester); PI(Propidium iodide); $\operatorname{DCF}\left(2^{\prime}, 7^{\prime}\right.$-dichlorofluorescein); DCFDA (dichlorofluorescein diacetate); $\triangle \Psi \mathrm{m}$ (mitochondrial membrane potential); PVDF (polyvinylidene difluoride); TBST (Tween/TBS); lv5-shRNA (lentiviral shRNA).

\section{Acknowledgements}

We thank Prof. Linfu Zhou and their lab members from Zhejiang University for constructive discussions, input and technical assistance.

\section{Funding Source}

This work was supported by the National Natural Science Foundation of Zhejiang Province (grant number LY14H020004) and by grants of Zhejiang Province Human Resources and Social Security Bureau, Health and Family Planning Commission of Zhejiang Province (grant number 2013KYA078).

\section{Disclosure Statement}

There is no conflict of interest.

\section{References}

1 Halestrap AP, Clarke SJ, Javadov SA: Mitochondrial permeability transition pore opening during myocardial reperfusion--a target for cardioprotection. Cardiovasc Res 2004;61:372-385. 


\section{Cellular Physiology Cell Physiol Biochem 2016;39:2121-2134

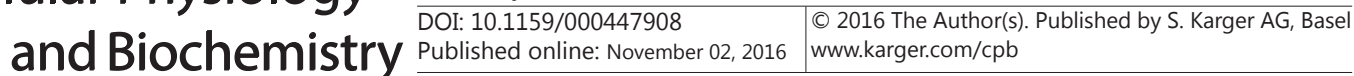 \\ Gao et al.: Targeting Dynamin 2}

2 Liu H, Mao P, Wang J, Wang T, Xie CH: Allicin Protects PC12 Cells Against 6-OHDA-Induced Oxidative Stress and Mitochondrial Dysfunction via Regulating Mitochondrial Dynamics. Cell Physiol Biochem 2015;36:966979.

3 Frank S, Gaume B, Bergmann-Leitner ES, Leitner WW, Robert EG, Catez F, Smith CL, Youle RJ: The role of dynamin-related protein 1, a mediator of mitochondrial fission, in apoptosis. Dev Cell 2001;1:515-525.

4 Hausenloy DJ, Yellon DM: The mitochondrial permeability transition pore: its fundamental role in mediating cell death during ischaemia and reperfusion. J Mol Cell Cardiol 2003;35:339-341.

5 Ong SB, Subrayan S, Lim SY, Yellon DM, Davidson SM, Hausenloy DJ: Inhibiting mitochondrial fission protects the heart against ischemia/reperfusion injury. Circulation 2010;121:2012-2022.

6 Sharp WW: Dynamin-related protein 1 as a therapeutic target in cardiac arrest. J Mol Med 2015;93:243252.

7 Ferguson SM, De Camilli P: Dynamin, a membrane-remodelling GTPase. Nat Rev Mol Cell Biol 2012;13:7588.

8 Kirchhausen T, Macia E, Pelish HE: Use of dynasore, the small molecule inhibitor of dynamin, in the regulation of endocytosis. Methods Enzymol 2008;438:77-93.

9 Gao DC, Zhang L, Dhillon R, Hong TT, Shaw RM, Zhu JH: Dynasore protects mitochondria and improves cardiac lusitropy in langendorff perfused mouse heart. PLoS One 2013;8:e60967.

10 Zanoteli E, Vergani N, Campos Y, Vainzof M, Oliveira ASB, d'Azzo A: Mitochondrial Alterations In Dynamin 2-Related Centronuclear Myopathy. Arquivos De Neuro-Psiquiatria 2009;67:102-104.

11 Ehler E, Moore-Morris T, Lange S: Isolation and culture of neonatal mouse cardiomyocytes. J Vis Exp DOI: 10.3791/50154.

12 Rubinson DA, Dillon CP, Kwiatkowski AV, Sievers C, Yang L, Kopinja J, Rooney DL, Zhang M, Ihrig MM, McManus MT, Gertler FB, Scott ML, Van Parijs L: A lentivirus-based system to functionally silence genes in primary mammalian cells, stem cells and transgenic mice by RNA interference. Nat Genet 2003;33:401406.

13 Qu Y, Karnabi E, Chahine M, Vassalle M, Boutjdir M: Expression of skeletal muscle Na(V)1.4 Na channel isoform in canine cardiac Purkinje myocytes. Biochem Biophys Res Commun 2007;355:28-33.

14 Karnabi E, Qu Y, Mancarella S, Yue Y, Wadgaonkar R, Boutjdir M: Silencing of Cav1.2 gene in neonatal cardiomyocytes by lentiviral delivered shRNA. Biochem Biophys Res Commun 2009;384:409-414.

15 Zhang N, Wang S, Li Y, Che L, Zhao Q: A selective inhibitor of Drp1, mdivi-1, acts against cerebral ischemia/ reperfusion injury via an anti-apoptotic pathway in rats. Neurosci Lett 2013;535:104-109.

16 Chen H, Detmer SA, Ewald AJ, Griffin EE, Fraser SE, Chan DC: Mitofusins Mfn1 and Mfn2 coordinately regulate mitochondrial fusion and are essential for embryonic development. J Cell Biol 2003;160:189-200

17 Huser J, Rechenmacher CE, Blatter LA: Imaging the permeability pore transition in single mitochondria. Biophys J 1998;74:2129-2137.

18 Duchen MR: Mitochondria and Ca(2+)in cell physiology and pathophysiology. Cell Calcium 2000;28:339348.

19 Du W, Pan Z, Chen X, Wang L, Zhang Y, Li S, Liang H, Xu C, Zhang Y, Wu Y, Shan H, Lu Y: By targeting Stat3 microRNA-17-5p promotes cardiomyocyte apoptosis in response to ischemia followed by reperfusion. Cell Physiol Biochem 2014;34:955-965.

20 Ruan Y, Dong C, Patel J, Duan C, Wang X, Wu X, Cao Y, Pu L, Lu D, Shen T, Li J: SIRT1 suppresses doxorubicininduced cardiotoxicity by regulating the oxidative stress and p38MAPK pathways. Cell Physiol Biochem 2015;35:1116-1124.

21 Kowaltowski AJ, Castilho RF, Vercesi AE: Mitochondrial permeability transition and oxidative stress. FEBS Lett 2001;495:12-15.

22 Zweier JL, Flaherty JT, Weisfeldt ML: Direct measurement of free radical generation following reperfusion of ischemic myocardium. Proc Natl Acad Sci U S A 1987;84:1404-1407.

23 Pendyala S, Usatyuk PV, Gorshkova IA, Garcia JGN, Natarajan V: Regulation of NADPH Oxidase in Vascular Endothelium: The Role of Phospholipases, Protein Kinases, and Cytoskeletal Proteins. Antioxid Redox Signal 2009;11:841-860.

24 Moujalled DM, Cook WD, Murphy JM, Vaux DL: Necroptosis induced by RIPK3 requires MLKL but not Drp1. Cell Death Dis 2014;5:e1086.

25 Reddy PH: Inhibitors of Mitochondrial Fission as a Therapeutic Strategy for Diseases with Oxidative Stress and Mitochondrial Dysfunction. J Alzheimers Dis 2014;40:245-256. 


\section{Cellular Physiology Cell Physiol Biochem 2016;39:2121-2134 \begin{tabular}{ll|l} 
DOI: 10.1159/000447908 & $\begin{array}{l}\text { O 2016 The Author(s). Published by S. Karger AG, Basel } \\
\text { www.karger.com/cpb }\end{array}$
\end{tabular} \\ Gao et al.: Targeting Dynamin 2}

26 Gilleron J, Carette D, Fiorini C, Dompierre J, Macia E, Denizot JP, Segretain D, Pointis G: The large GTPase dynamin2: A new player in connexin 43 gap junction endocytosis, recycling and degradation. Int J Biochem Cell Biol 2011;43:1208-1217.

27 Brady NR, Hamacher-Brady A, Gottlieb RA: Proapoptotic BCL-2 family members and mitochondrial dysfunction during ischemia/reperfusion injury, a study employing cardiac HL-1 cells and GFP biosensors. Biochim Biophys Acta 2006;1757:667-678.

28 Chan S, Chan GC, Ye J, Lian Q, Chen J, Yang M: Thrombopoietin Protects Cardiomyocytes from Iron-Overload Induced Oxidative Stress and Mitochondrial Injury. Cell Physiol Biochem 2015;36:2063-2071.

29 Singleton PA, Pendyala S, Gorshkova IA, Mambetsariev N, Moitra J, Garcia JGN, Natarajan V: Dynamin 2 and c-Abl Are Novel Regulators of Hyperoxia-mediated NADPH Oxidase Activation and Reactive Oxygen Species Production in Caveolin-enriched Microdomains of the Endothelium. J Biol Chem 2009;284:34964-34975.

30 Zamora PL, Villamena FA: Pharmacological approaches to the treatment of oxidative stress-induced cardiovascular dysfunctions. Future Med Chem 2013;5:465-478.

31 McNiven MA, Cao H, Pitts KR, Yoon Y: The dynamin family of mechanoenzymes: pinching in new places. Trends Biochem Sci 2000;25:115-120.

32 Orth JD, McNiven MA: Dynamin at the actin-membrane interface. Curr Opin Cell Biol 2003;15:31-39.

33 Schafer DA: Regulating actin dynamics at membranes: a focus on dynamin. Traffic 2004;5:463-469.

34 Li J, Xu L, Ye J, Li X, Zhang D, Liang D, Xu X, Qi M, Li C, Zhang H, Wang J, Liu Y, Zhang Y, Zhou Z, Liang X, Li J, Peng L, Zhu W, Chen YH: Aberrant dynamin 2-dependent $\mathrm{Na}(+) / \mathrm{H}(+)$ exchanger-1 trafficking contributes to cardiomyocyte apoptosis. J Cell Mol Med 2013;17:1119-1127.

35 Gilleron J, Carette D, Fiorini C, Dompierre J, Macia E, Denizot JP, Segretain D, Pointis G: The large GTPase dynamin2: a new player in connexin 43 gap junction endocytosis, recycling and degradation. Int J Biochem Cell Biol 2011;43:1208-1217.

36 Fish KN, Schmid SL, Damke H: Evidence that dynamin-2 functions as a signal-transducing GTPase. J Cell Biol 2000;150:145-154.

37 Kashiwakura Y, Watanabe M, Kusumi N, Sumiyoshi K, Nasu Y, Yamada H, Sawamura T, Kumon H, Takei K, Daida H: Dynamin-2 regulates oxidized low-density lipoprotein-induced apoptosis of vascular smooth muscle cell. Circulation 2004;110:3329-3334.

38 Macia E, Ehrlich M, Massol R, Boucrot E, Brunner C, Kirchhausen T: Dynasore, a cell-permeable inhibitor of dynamin. Dev Cell 2006;10:839-850. 